\title{
Atypical Presentations of Laryngeal Cancers: Diagnostic and Treatment Dilemmas
}

\author{
1Jyoti Dabholkar, ${ }^{2}$ Neeti Madan Kapre, ${ }^{3}$ Ameya Bihani
}

\begin{abstract}
Introduction: Larynx is a complex organ with various subsites and a myriad of pathologies. Atypical presentations of laryngeal cancers are rare with literature only in the form of case reports or series with small number of patients.
\end{abstract}

Aim of study: It is important to recognize rare presentations of laryngeal carcinomas in order to pre-empt misdiagnosis and execute an appropriate treatment plan.

Materials and methods: We present a case series of four such atypical presentations which were managed with precise and individualized approaches.

Results: Three patients with rare manifestations of laryngeal squamous cancers were treated surgically in view of gross exolaryngeal spread. Single case of melanoma was treated with chemoradiation followed by surgical salvage.

Conclusion: All four patients were identified through adequate diagnostic precision on part of the clinician.

Clinical implications: The form and function of the larynx are crucial to the quality of life of a patient. Laryngeal carcinomas require appropriate treatment approach to ensure the same. The clinician needs to be aware of these atypical presentations as well in order to diagnose and treat these patients effectively.

Keywords: Atypical, Cancers, Larynx.

How to cite this article: Dabholkar J, Kapre NM, Bihani A. Atypical Presentations of Laryngeal Cancers: Diagnostic and Treatment Dilemmas. Int J Phonosurg Laryngol 2015;5(2):53-56.

Source of support: Nil

Conflict of interest: None

\section{INTRODUCTION}

Larynx is a complex organ with several subsites. Each can present with a myriad of pathologies and distinct clinical manifestations. The form and function of the larynx is of utmost importance for a sound quality of life. Hence, management of rare laryngeal lesions poses a formidable challenge to the physician. A high index of suspicion for

${ }^{1}$ Professor and Head, ${ }^{2}$ Senior Clinical Fellow, ${ }^{3}$ Senior Registrar

${ }^{1-3}$ Department of ENT, Head and Neck Surgery, KEM Hospital Mumbai, Maharashtra, India

Corresponding Author: Neeti Madan Kapre, Senior Clinical Fellow, Department of ENT, Head and Neck Surgery, KEM Hospital, Mumbai, Maharashtra, India, Phone: 24107000 , e-mail: hi.neeti@gmail.com diagnosis and knowledge of the biological behavior of these lesions are imperative to ensure optimum outcomes.

A small percentage of laryngeal lesions are found to be atypical on routine laryngeal examination either due to appearance, location or histology. ${ }^{1}$ Here, we present a series of cases with involvement of larynx by rare lesions. We have also discussed their management alongwith with relevant review of literature.

\section{CASE REPORTS}

\section{Case 1}

\section{Spindle Cell Carcinoma of the Larynx}

A 52-year-old gentleman presented with complaints of hoarseness of voice since 1 month. Indirect laryngoscopy revealed an anterior commissure pedunculated mass. Both vocal cords were mobile. Microlaryngoscopy revealed a proliferative polypoidal mass over the anterior commissure. Repeated biopsies suggested a dysplastic epithelium and occasional spindle cells without evidence of malignancy. Contrast-enhanced computed tomography $(\mathrm{CT})$ revealed the lesion over anterior commissure with erosion of thyroid cartilage and escape of tumor through the Broyle's ligament with exolaryngeal spread (Fig. 1). Within a short span of 10 days, patient had significant progression of disease with obstructive symptoms and had to undergo a tracheostomy. Repeat imaging revealed bilateral involvement of glottis and

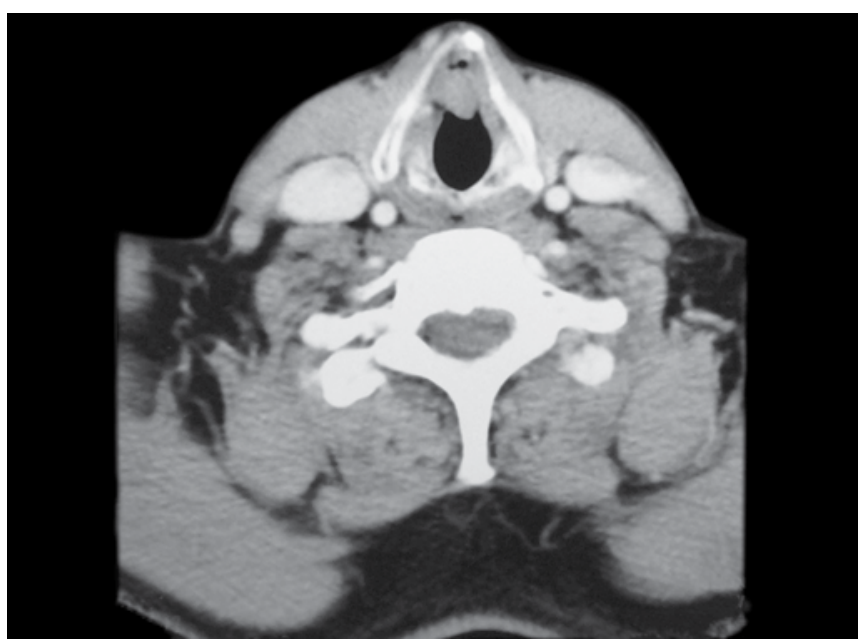

Fig. 1: Spindle cell carcinoma presenting as a anterior commissure polypoidal growth 
subglottic extension with erosion of thyroid cartilage. Computed tomography-guided fine-needle aspiration cytology was attempted through this anterior soft tissue component of the disease. This confirmed the diagnosis of sarcomatoid carcinoma. Patient was staged at T4N0M0 in view of exolaryngeal spread without nodal or distant metastases. He underwent a total laryngectomy with bilateral neck dissection and primary tracheoesophageal prosthesis insertion. Final histopathology showed pT4N0, and hence patient was advised adjuvant radiation.

Spindle cell carcinoma is a highly malignant variant of squamous carcinoma and comprises 2 to $3 \%$ of laryngeal cancers. ${ }^{2}$ It is considered to be a biphasic tumor that is composed of a squamous cell carcinoma (in situ or invasive) and spindle cell carcinoma with sarcomatous appearance. ${ }^{3}$ Therefore, diagnosis often poses a challenge. It most commonly affects the glottis in the majority of cases $(70 \%)$ and lesions are often polypoidal or pedunculated. ${ }^{4}$ The staging and treatment remains the same as squamous carcinoma. Laser excision and organ preserving surgeries are an acceptable option for treatment for early lesions. Radiation and chemotherapy are effective modalities of adjuvant treatment. Survival outcomes of this rare variant are good with poor prognostic factors being higher stages, large tumors $(3 \mathrm{~cm})$ with a predominance of epithelial component, nonglottic tumors, fixed vocal cords, history of radiotherapy and metastasis to regional lymph nodes and distant metastasis. ${ }^{4}$

\section{Case 2}

\section{Distinct Coexisting Thyroid and Laryngeal Pathologies}

There have been reports of coexisting neoplasms in the thyroid gland of different pathological origin, the so-called collision tumors. ${ }^{5}$ However, there has not been much-reported in literature about coexisting thyroid and laryngeal neoplasms.

A 45-year-old lady presented with complaints of hoarse voice and swelling in front of the neck. On laryngeal examination, an ulceroproliferative growth was seen on the ipsilateral side of the neck mass, with fixity of the hemilarynx. Imaging revealed an enhancing thyroid mass involving both lobes of thyroid, right more than left, with intralaryngeal extension across the thyroid cartilage and through the cricothyroid membrane. Fineneedle aspiration cytology of the swelling reported a follicular neoplasm, Bethesda category IV. Decision was taken to proceed with a total thyroidectomy with bilateral central and lateral compartment neck dissection and total laryngectomy with primary pharyngeal reconstruction with primary tracheoesophageal prosthesis insertion after due counseling of the patient about the rare presentation of the disease. The final histopathology showed laryngeal squamous cell carcinoma with focal cartilage involvement pT3 and metastases to regional lymph nodes. There was no involvement of the thyroid soft tissue. The lesion in the thyroid was a separate follicular adenoma. Patient received adjuvant radiation treatment as warranted and is voicing well with the voice prosthesis.

\section{Case 3}

\section{Distinct Coexisting Thyroid and Laryngeal Pathologies}

A 52-year-old female presented with history of gradually progressive swelling in front of the neck since 2 years with difficulty in swallowing for the past 2 months. Clinical examination showed multinodular thyroid swelling. Flexible fiber optic examination revealed a supraglottic growth mainly involving the epiglottis and anterior parts of the aryepiglottic growth. Both vocal cords were mobile. Fine-needle aspiration cytology from the thyroid swelling was reported as papillary thyroid carcinoma, whereas biopsy from the supraglottic mass was squamous cell carcinoma. Contrast-enhanced CT imaging demonstrated an enhancing soft tissue mass over the supraglottis without invasion of the pre-epiglottic, paraglottic spaces or laryngeal cartilages. The thyroid nodule was $3.3 \mathrm{~cm}$ in largest dimension with evidence of calcification within and enhancing on the scan. She was planned to undergo a total thyroidectomy with central compartment clearance with bilateral selective neck dissection and microlaryngoscopic laser excision of the supraglottic mass (Figs 2 to 4). The final pathological staging was pT2N0 for the laryngeal lesion and pT3N1b for the thyroid. The patient had a satisfactory postoperative recovery.

The first impression that any clinician would routinely make in this clinical scenario would be a thyroid carcinoma with intralaryngeal extension or a laryngeal

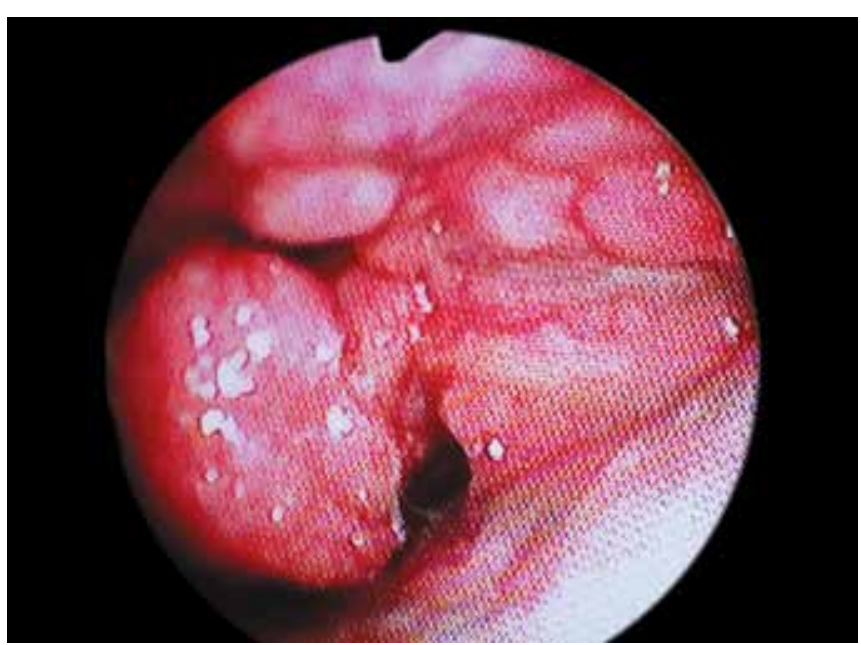

Fig. 2: Laryngoscopic picture of an epiglottic mass coexisting with thyroid pathology 


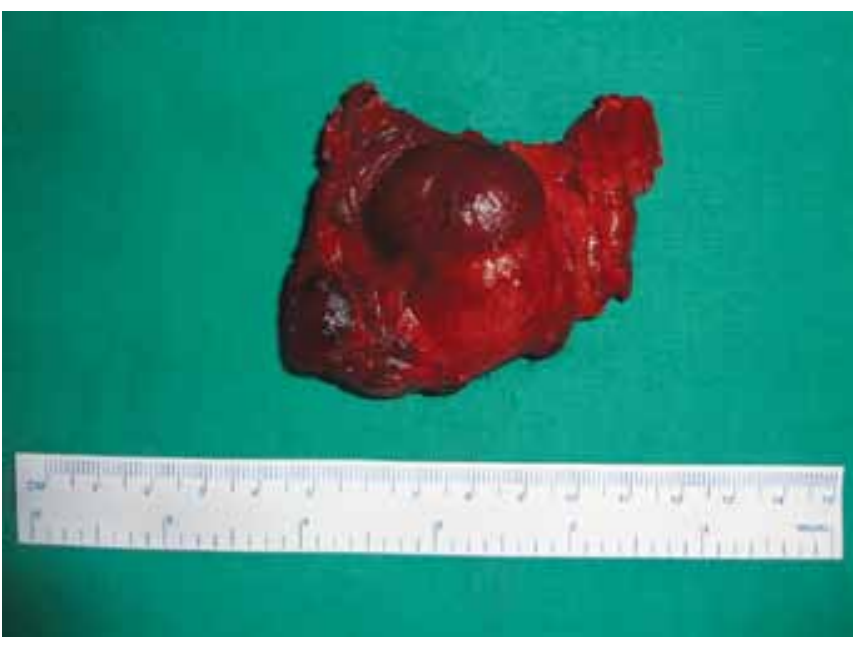

Fig. 3: Specimen of total thyroidectomy

pathology with exolaryngeal spread into the thyroid. It was very rare and interesting to note two dissimilar pathologies with distinctly different malignant potentials and yet coexisting in such close vicinity. Literature reports chondrosarcomas of the larynx presenting with thyroid masses undergoing a similar line of management. ${ }^{6}$ However, no solid data exist for coexisting squamous carcinoma of larynx with distinct but coexisting benign or malignant thyroid masses.

\section{Case 4}

\section{Primary Malignant Melanoma of the Larynx}

This was a 38-year-old gentleman with hoarse voice and difficulty in swallowing. He had undergone an emergency tracheostomy elsewhere for difficulty in breathing. Direct laryngoscopy showed a transglottic black-blue mass occluding the glottis inlet with significant subglottic extension. Hypopharynx was uninvolved. Biopsy confirmed the diagnosis of mucosal melanoma with immunohistochemistry positive for S-100 and HMB-45. Positron emission tomography (PET) scan was done in view of the aggressive nature and large volume of the disease. Positron emission tomography-computed tomography showed uptake in the larynx with metastatic uptake in the cervical lymph nodes (Fig. 5). Patient received curative doses of chemoradiation (70 Gy in 35 fractions over 7 weeks with 7 cycles of cisplatin) with a view for organ preservation. ${ }^{7}$ Patient had persistent residual disease after treatment and warranted salvage surgery. He subsequently underwent total laryngectomy with primary closure and primary tracheoesophageal puncture. Patient is disease free after a follow-up of more than 24 months at present. Although systemic chemotherapeutic agents have had disappointing results in the treatment of melanomas, in view of his young age he was advised a cisplatinum-based chemotherapy regimen.

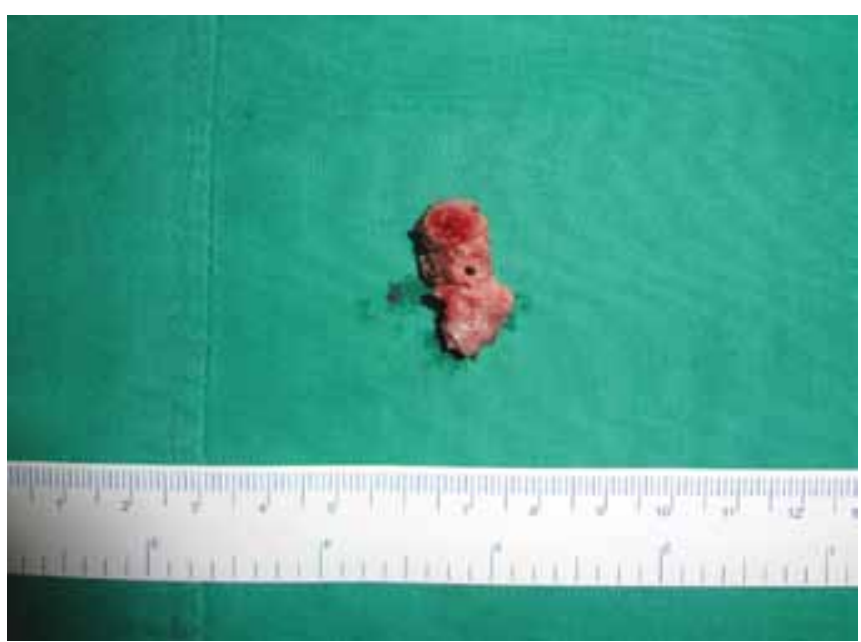

Fig. 4: Separate specimen of the epiglottic growth laser excision

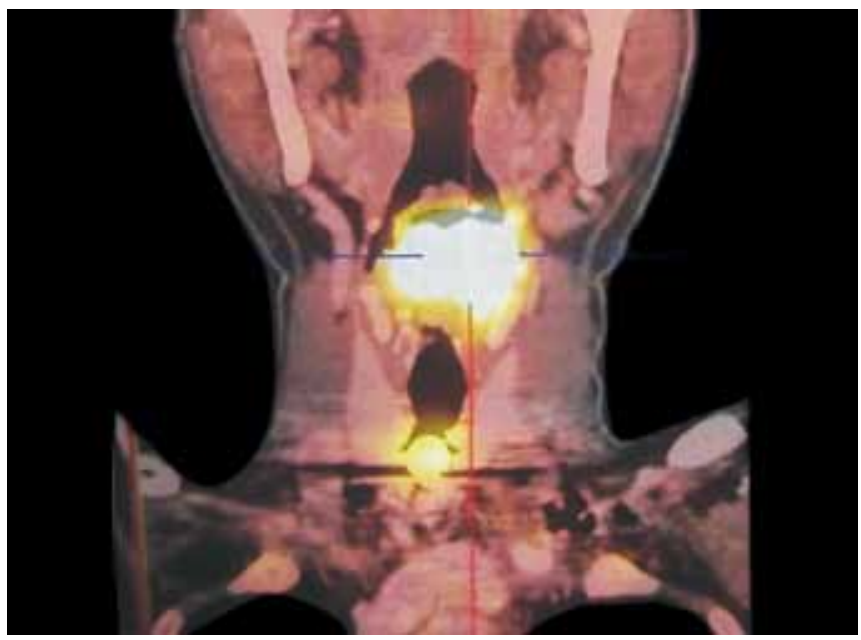

Fig. 5: Positron emission tomography CT image of malignant melanoma of the subglottis

Primary malignant melanoma of the larynx is a very rare entity with less than 60 cases reported in literature. Laryngeal malignant melanoma constitutes only 3.8 to $7.4 \%$ of mucosal melanomas. ${ }^{8}$ Most of the mucosal melanomas have distant metastases at presentation. ${ }^{9,10}$ Confirmation of diagnosis requires histopathological examination and immunohistochemistry. According to Allen and Spitz, ${ }^{11}$ malignant melanocytes must be identified in the dermoepidermal junction or in the surface epithelium (i.e. in situ component) to establish a diagnosis of primary malignant melanoma. Treatment is primarily surgical excision with adjuvant treatment. Adjuvant radiation improves local control; however, it does not necessarily translate into improved prognosis. ${ }^{12}$

\section{REFERENCES}

1. McCoy G. Atypical laryngeal lesions-problems in diagnosis. Calif Med 1954 Nov;81(5):328-331.

2. Völker H-U, Scheich M, Holler S, Strobel P, Hagen R, MullerHermelink HK, Eck M. Differential diagnosis of laryngeal spindle cell carcinoma and inflammatory myofibroblastic 
tumor-report of two cases with similar morphology. Diagnostic Pathol 2007 Jan;2:1.

3. Katase N, Tamamura R, Gunduz M, Murakami J, Asaumi J, Tsukamoto G, Sasaki A, Nagatsuka H. A spindle cell carcinoma presenting with osseous metaplasia in the gingiva: a case report with immunohistochemical analysis. Head Face Med 2008 Dec 1;4:28.

4. Thompson LD, Wieneke JA, Miettinen M, Heffner DK. Spindle cell (sarcomatoid) carcinomas of the larynx: a clinicopathologic study of 187 cases. Am J Surg Pathol 2002 Feb;26(2):153-170.

5. Takano K, Kikuchi K, Matsumiya H, Himi T. Collision tumour of the thyroid: follicular carcinoma plus papillary carcinoma plus adenomatous goitre. World J Med Surg 2013;4(2):5.

6. Pignataro L, Peri A, Pagani D, Iudica F, Scaramellini G. Cricoid chondrosarcoma coexisting with a thyroid mass: case report and review of the literature. Tumori 2006 MayJun;92(3):257-259.

7. Medina JE, Ferlito A, Pellitteri PK, Shaha AR, Khafif A, Devaney KO, Fisher SR, O'Brien CJ, Byers RM, Robbins KT, et al. Current management of mucosal melanoma of the head and neck. J Surg Oncol 2003 Jun;83(2):116-122.

8. Snow GB, van der Esch EP, van Slooten EA. Mucosal melanomas of the head and neck. Head Neck Surg 1978 Sep-Oct;1(1):24-30.

9. Zaghi S, Pouldar D, Lai C, Chhetri DK. Subglottic presentation of a rare tumor: primary or metastatic? Primary mucosal melanoma of the subglottic larynx. JAMA Otolaryngol Head Neck Surg 2013 Jul;139(7):739-740.

10. Sirikanjanapong S, Lanson B, Amin M, Martiniuk F, Kamino H, Wang BY. Collision tumor of primary laryngeal mucosal melanoma and invasive squamous cell carcinoma with IL-17A and CD70 gene over-expression. Head Neck Pathol 2010 Dec;4(4):295-299.

11. Allen AC, Spitz S. Malignant melanoma: a clinicopathological analysis of the criteria for diagnosis and prognosis. Cancer 1953 Jan;6(1):1-45.

12. Saigal K, Weed DT, Reis IM, Markoe AM, Wolfson AH, Nguyen-Sperry J. Mucosal melanomas of the head and neck: the role of postoperative radiation therapy. ISRN Oncol 2012;2012:785131. 